\title{
ECG Evaluation Not Performed Due to Poor Quality of Printed ECG
}

National Cancer Institute

\section{Source}

National Cancer Institute. ECG Evaluation Not Performed Due to Poor Quality of Printed

ECG. NCI Thesaurus. Code C114180.

A printed electrocardiographic recording with poor printout quality (e.g. a faded paper ECG tracing or ECG with inconsistent printing speed) such that measurements and/or interpretation cannot be performed. (CDISC) 